\title{
Metacognição e resoluÇão \\ DE PROBLEMAS ARITMÉTICOS VERBAIS: \\ TEORIA E IMPLICAÇÕES PEDAGÓGICAS
}

Marcelo Chahon ${ }^{\star}$

\section{Resumo}

O presente trabalho propõe uma revisão do constructo psicológico de metacognição, especialmente enquanto habilidade utilizada em atividades de resolução de problemas e especificamente com relação a problemas aritméticos verbais. Procura-se evidenciar a complexidade dos modelos de desenvolvimento da competência em representar e resolver diferentes problemas envolvendo as quatro operações, ressaltando as implicações pedagógicas sugeridas por ampla literatura. Finalmente, conforme as idéias do Professor Franco Lo Presti Seminerio, é vislumbrada na metacognição um recurso psicopedagógico fundamental à aquisição de autonomia cognitiva pelo jovem aprendiz, cuja eficácia pôde ser observada em sucessivas intervenções experimentais conduzidas pelo Laboratório de Metacognição da UFRJ.

Palavras-chave: Metacognição. Resolução de problemas. Educação Matemática. Psicopedagogia.

\section{Metacognition AND VERBAL ARITHMETIC PROBLEM SOLVING:}

\section{THEORY AND PEDAGOGICAL IMPLICATIONS}

\begin{abstract}
The present work proposes a review of the psychological metacognition construct, specially as a skill used in problem solving activities, and specifically in relation to verbal arithmetic problems. It is here attempted to evidence the complexity of the development models regarding the competence in representing and solving different problems involving the four mathematical operations, emphasizing the

* Doutor em Psicologia / UFRJ (área de concentração: Desenvolvimento Cognitivo) Psicólogo da Secretaria Municipal de Saúde de Petrópolis. Rua Raul Pompéia, 190 / 901 - Copacabana - Rio de Janeiro-RJ Formação e Licenciatura em Psicologia pela UFRJ; Mestrado e Doutorado em Psicologia pela UFRJ (área de concentração: Desenvolvimento Cognitivo); Psicólogo da Secretaria Municipal de Saúde de Petrópolis desde 03/2000; Especialização em Psiquiatria e Psicoterapia da Infância (Instituto de Psiquiatria / UFRJ); Especialização em Psicopedagogia na Educação (Faculdade de Educação / UFRJ).

E-mail: psichahon@hotmail.com
\end{abstract}


pedagogical implications suggested by literature. In accordance to the ideas of Professor Franco Seminerio, metacognition is seen as a fundamental psychopedagogical resource to the acquisition of cognitive autonomy by the young apprentice, which effectiveness was observed in experimental interventions conducted by UFRJ Metacognition Laboratory.

Keywords: Metacognition. Problem solving. Mathematical education. Psychopedagogy.

\section{Metacognição: "UM nOVo PARAdigma"}

Flavell define "metacognição" enquanto "qualquer conhecimento ou atividade cognitiva que toma como seu objeto, ou regula, qualquer aspecto de qualquer iniciativa cognitiva" (FLAVELL et al., 1999, p. 125). O "conhecimento metacognitivo" é subdividido pelo autor em três categorias interligadas: sobre pessoas, sobre tarefas e sobre estratégias. Em poucas palavras, diante de atividades de resolução de problemas, o conhecimento pelo aprendiz dos próprios recursos e a compreensão das exigências da tarefa levam ao emprego de procedimentos de resolução mais (ou menos) apropriados, inclusive ao reconhecimento (dito primário) da própria ignorância e a um conseqüente adiamento da tarefa.

Notadamente, Flavell sustenta que as habilidades metacognitivas infantis, envolvendo a capacidade de monitorar (de saber localizar-se em relação à sua meta) e auto-regular (planejar e avaliar) seu comportamento, se desenvolvem desde os 7 anos e podem ser ensinadas dentro do currículo escolar.

Bruner (2001, p. 63) situa os estudos sobre a metacognição como uma linha de pesquisa que valoriza a criança como construtora de um modelo do mundo, o qual é aprimorado por meio do intercâmbio subjetivo com o adulto ou com seus pares. Outras linhas de pesquisa aproximadas, segundo Bruner, incluem o desenvolvimento precoce da intersubjetividade humana nas relações mãe-bebê, a "teoria da mente" infantil, isto é, a compreensão das crenças e desejos dos outros, evidenciada ao menos a partir dos 4 anos, e investigações sobre aprendizagem colaborativa e resolução de problemas.

Segundo Seminerio et al. (1999), o conceito de metacognição estabelece uma ruptura frente ao paradigma behaviorista, levando ao tratamento dos processos mentais em primeira (e não em terceira) pessoa. A noção de "sentimento" de conhecer (feeling of knowing: FOK) formulada por Hart em 1965, sob a orientação de Flavell, como um julgamento (favorável ou não) que sucede ao ato de conhecer, ilustra de modo excelente essa oposição.

A crescente atividade experimental sobre o assunto, de caráter predominante diagnóstico, pode ser dividida entre o exame do conhecimento metacognitivo "mais estável", desde os estudos sobre metamemória empreendidos por Flavell na década de 70, sob influência das concepções piagetianas de desenvolvimento cognitivo, e trabalhos conduzidos por Brown em torno das habilidades de regulação 
(mais sujeitas às influências do contexto), com apoio na tradição do processamento de informação (PAULA, 2001).

\section{RESOlUÇÃO DE PROBLEMAS: ASPECTOS GERAIS}

A resolução de problemas abrange um campo de investigação excepcionalmente amplo na história da psicologia cognitiva em particular, assim como na história (e filosofia) da matemática, traduzindo desde as últimas décadas do século XX mudanças profundas no campo pedagógico. Silva (1999) identifica uma maior ênfase às atividades de resolução de problemas junto ao declínio da chamada Matemática Moderna (1960-1970), fundamentada em uma perspectiva logicista tradicional, em favor de uma concepção heurística que, em síntese, valoriza o ensino de estratégias e processos de resolução como meio de promover o pensamento maduro e antecipar a formalização dos conceitos envolvidos. Justamente, as contribuições do construtivismo e das teorias de processamento de informação têm se revelado, no cenário brasileiro, as mais valiosas à orientação de um ensino "através da resolução de problemas" (ONUCHIC, 1999).

A posição construtivista, bastante divulgada na área de ensino de matemática por meio dos trabalhos de Constance Kamii (1989, 1993), privilegia a preservação da autonomia social, moral e intelectual da criança, uma vez que os conceitos aritméticos elementares são assinalados como produtos internos das primeiras construções de relações entre objetos, processo de abstração reflexiva (ou construtiva) que caracteriza o conhecimento estritamente lógico-matemático. A contrapartida pedagógica conduz assim à importância da (re) invenção e da troca de pontos de vista entre as crianças, com reflexos evidentemente positivos na confiança das mesmas em seu próprio raciocínio.

A abordagem do processamento de informação pode ser situada como uma nova linguagem construtora de "modelos" específicos da atividade cognitiva humana, surgida nas décadas de 50 e 60 em razão das exigências de adaptação a novas tecnologias (WOOD, 1996). A ênfase é aqui traduzida em uma avaliação minuciosa do domínio (ou perícia) do "resolvedor de problemas auto-suficiente", na expressão de Bruner, descrevendo os "metacomponentes" que se encontram associados aos processos de aquisição da informação e desempenho. Para Sternberg (2000, p. 309), em síntese, os estudantes "peritos" (experts) "tendem a dedicar mais dos seus recursos mentais ao planejamento global (visão geral) do que os solucionadores de problemas iniciantes", fazendo uso variado de estratégias informais ou "atalhos mentais" (heurísticas), com vistas a reduzir o espaço do problema dentro da memória de trabalho.

Mayer (1992), na tentativa de explicar a "capacidade para a matemática" segundo a mesma abordagem, analisou as operações mentais e os conhecimentos que atuam como componentes principais em atividades de resolução de problemas. Os "passos" necessários descritos incluem: a conversão do problema em uma "representação" interna, produto de sua tradução apropriada em termos lingüísticos e factuais, e de sua integração em um tipo de categorização esquemática 
(semântica); a "solução" do problema, com a aplicação das operações matemáticas legítimas àquela representação resultando de um planejamento estratégico (incluindo a capacidade de monitorar o plano de solução) e de sua execução em termos algorítmicos (desde modelos de contagem desenvolvidos precocemente).

Claramente interligados a estes passos, Davidson et al. (1996) apontaram quatro processos metacognitivos fundamentais que orientam a resolução de problemas matemáticos: identificar e definir o problema; representar mentalmente o problema; planejar como proceder; e por fim avaliar o próprio desempenho.

Para Brito (2000), refletindo na solução de problemas enquanto "metodologia de ensino", as maiores dificuldades parecem se concentrar na etapa de sua representação, levando à crítica de um ensino geralmente focalizado na resolução de problemas rotineiros por meio de fórmulas e modelos estereotipados, em prejuízo da habilidade de transferir situações escolares para a vida e de uma desejável generalização algorítmica dos procedimentos que conduzem à solução de um problema.

\section{Problemas aditivos e subtrativos: Teoria e Pesquisa}

Tomando por eixo a excelente síntese de Nunes e Bryant (1997), em cujo livro diferentes conteúdos matemáticos são analisados segundo a teoria do "campo conceitual" de Vergnaud, verifica-se que a compreensão infantil dos conceitos de adição e subtração se dá por meio do domínio de um número crescente de situações-problema, o qual deriva da utilização de uma variedade de procedimentos, baseados em invariantes (ou propriedades) diferentes "teoremas em ato" e sustentados por múltiplos sistemas de sinais (representações simbólicas).

Em geral, a literatura distingue quatro grandes categorias de problemas verbais mais simples: de transformação, combinação, comparação e igualamento. Vergnaud (apud FAYOL, 1996) propõe uma classificação parcialmente distinta conforme as "operações de pensamento" ou o "cálculo relacional" envolvido, a partir de três conceitos fundamentais: medida, transformação no tempo e relacionamento (VASCONCELOS, 1998; VERGNAUD, 1986).

As relações semânticas (FAYOL, 1996), ao definir as categorias acima pelas situações que descrevem) postas em jogo permitem entrever uma maior facilidade na representação e resolução das duas primeiras categorias de enunciado acima referidas, ao passo que os problemas de comparação, envolvendo a quantificação de uma relação estática, são via de regra mais complexos uma vez que sua solução exige, simultaneamente, a compreensão de relações parte-todo e da relação inversa entre adição e subtração. Contudo, também a forma de apresentação dos problemas, ainda que apenas considerando textos convencionais, pouco extensos, acarreta importantes diferenças de desempenho. A posição da incógnita, em especial, pode tornar um problema de transformação mais difícil desde que se afasta do resultado rumo ao termo inicial, e um problema de combinação torna-se igualmente mais complicado de resolver quando uma das partes é desconhecida, exigindo a compreensão da subtração como operação inversa (FAYOL, 1996). 
Na sua maioria, os modelos teóricos que tratam do domínio dessas situações apóiam-se no conhecimento lógico-matemático infantil (VASCONCELOS, 1998), mas deve-se ter em conta o desenvolvimento das habilidades de compreensão da linguagem como fator determinante ao desempenho da criança, pela dificuldade em "mapear" determinadas formas lingüísticas no interior das estruturas conceituais de que a criança dispõe (CUMMINS et al., 1998).

Numerosos artigos em periódicos especializados procuram explicitar os processos subjacentes à representação e resolução de problemas aditivos e subtrativos, esboçando hipóteses sobre a psicogênese e o ensino dessas operações. Em geral os variados procedimentos experimentais revistos buscam predizer as variações de desempenho infantil devidas a deficiências de conhecimento matemático e semântico, fazendo uso das estratégias e erros, umas e outros cuidadosamente codificados, enquanto critério de validação do modelo proposto.

Um exemplo abrangente de intervenção, que conjuga algumas das implicações pedagógicas recorrentes em tais estudos, pode ser encontrado na "perspectiva metodológica da Resolução de Problemas", adotada por Smole e Diniz (2001). As autoras enfatizam o uso desejável dos recursos da oralidade (p.ex. a exposição dos procedimentos de resolução ou sua elaboração coletiva), do desenho (representando aspectos da situação ou sua solução completa) e da escrita (invenção de novos problemas com os mesmos dados ou com a mesma pergunta), a fim de privilegiar o confronto entre diferentes textos (convencionais ou não) e estruturas matemáticas, articulando a linguagem matemática ao domínio da língua materna. Este breve conjunto de idéias volta-se, naturalmente, também ao ensino das operações ditas mais complexas.

Fini (2001), de forma igualmente ampla, aponta para o equívoco em adiar o ensino de problemas aritméticos verbais até uma melhor aprendizagem das operações numéricas, uma vez que a construção da aritmética, na perspectiva piagetiana, resulta da "aritmetização lógica a partir de situações de sua vida cotidiana". De resto, é também reiterada na literatura a crítica a um ensino tradicional, pela ênfase excessiva no cálculo numérico e no uso de "palavras-chave", em detrimento da compreensão das relações entre os dados e da análise das diferenças semânticas, lógicas ou sintáticas entre os problemas. Ainda, a pesquisa psicológica evidencia um uso freqüentemente indiscriminado do material concreto em sala de aula, justificado em uma interpretação inadequada da teoria piagetiana acerca do papel atribuído à ação junto ao meio físico.

\section{Problemas de multiplicação e divisão: Teoria e Pesquisa}

Também estes problemas se distinguem semanticamente, seja em função da clareza da relação um-para-muitos no enunciado, como devido à quantidade que serve de incógnita (KOUBA, 1989). As situações multiplicativas mais simples trazem o produto desconhecido, e aquela relação se evidencia em um sentido de "agrupamento" (grouping) ou "combinação" (matching) - no primeiro caso a relação é facilmente representada de vez que um dos termos é identificado como simultaneamente um objeto em si e um recipiente que os agrupa. 
E para as situações de divisão, os mesmos sentidos se aplicam a problemas onde a incógnita se volta ao número de conjuntos "problemas de medida" (measurement), ou ao número de elementos em cada conjunto (cota) "problemas partitivos" (partitive).

Embora a representação direta dos primeiros envolva o estabelecimento de uma correspondência um-para-muitos entre cota e receptores, algo difícil até pelo menos 7 anos, podem induzir, porém a uma estratégia material de subtração repetida, não se justificando conceitualmente o predomínio maciço de problemas partitivos nos livros didáticos (SELVA, 1998).

Em uma categorização mais abrangente, estes problemas mais simples pertencem a uma classe comum de problemas que reúnem três fatores: dois números cardinais finitos, o multiplicando e o produto, e uma terceira medida "pura", sem dimensão, o multiplicador; o fator desconhecido determina quando se trata de uma situação multiplicativa ou de um problema de divisão de medida ou partitivo. Na classificação de Vergnaud (1988 apud BELL et al., 1989), tratam-se de problemas de "isomorfismo de medidas", estruturas assimétricas devido aos papéis distintos desempenhados pelas quantidades multiplicadas, envolvendo um número inteiro de repetições de um conjunto de objetos (ou de uma quantidade contínua). Em particular, duas das estruturas semânticas mais elementares, estruturas partetodo e de iteração, são similares a estruturas de adição e subtração correspondentes, parte-parte-todo (combinação) e de transformação.

Sobre as origens psicológicas das operações de multiplicação e divisão, as contribuições da epistemologia genética permitem identificar competências relacionais precoces que precedem a emergência gradual do "pensamento multiplicativo" na criança, levando Nunes e Bryant (1997) a sugerirem que a exploração desse conhecimento prévio seja capaz de favorecer a percepção da correspondência um-para-muito em problemas verbais, que exigem o cálculo de valores absolutos. No caso da divisão, os autores destacam a importância de ações cotidianas de distribuição (de material discreto) ou cortes (de material contínuo), no estabelecimento a princípio informal de uma relação inversa entre o número de partes e o tamanho das mesmas (cota), embora seja difícil na maioria dos experimentos, com crianças a partir de 7 anos, precisar se elas de fato captam as relações parte-todo (intensivas) implicadas em suas ações.

\section{Metacognição e aprendizagem Matemática}

Bruner foi provavelmente um dos primeiros a apresentar uma descrição "metaprocessual" da aprendizagem matemática, na medida em que, antes de tudo, a própria matemática é vista como "a metalinguagem mais geral até hoje criada, fornecendo as formas e estruturas que permitem compreender as regularidades da natureza" (BRUNER, 1976, p. 51). Para Bruner, a instrução comporta a "comunicação" dos processos subjacentes ao pensamento inteligente e adaptativo da criança, na forma de um domínio recompensador, uma "prontidão" ao uso de três sistemas paralelos (e inatos) de processamento informacional: através da ação (representação ativa), pelas transformações econômicas da organização perceptiva 
(representação icônica), e através da linguagem natural ou artificial (representação simbólica).

Tal perspectiva é de certo modo ampliada por Seminerio (1995a, 1995b), tratando da noção de metaprocesso enquanto "competência recursiva" inata que possibilita um controle intencional de invariâncias ou regras lógicas pertinentes a linguagens representativas "linguagens-código inatas" de ordem inferior. Fazendo uso de um referencial teórico abrangente, Seminerio investiga os passos filogenéticos que levaram à constituição do aparato cognitivo humano como sistema organizador da informação circundante. Em suma, a partir da linguagem discursiva humana é admitida uma "inferência generalizadora" desde os processos de diferenciação da informação encontrados no canal percepto-sensorial audiofonético, que se inscreve junto ao início da hominização, até processos isomórficos situados junto ao canal visomotor, filogeneticamente mais remoto.

Seminerio reconhece no ápice do canal visomotor as regras últimas da ação transformadora, tão bem analisadas por Piaget nas diversas aquisições que marcam o estágio operatório concreto do desenvolvimento cognitivo. Contudo, o construtivismo piagetiano é aqui reinterpretado em escala filogenética, ocupando a ação um caráter estrutural na emergência das linguagens e suas regras mentais correspondentes. Apoiado em argumentos como o proposto por Chomsky acerca da gramática de uma língua, cujo domínio precoce parece pressupor regras universais altamente restritivas, e refletindo em torno da emergência das operações lógicas superiores definidas por Piaget, Seminerio (1984, 1994) questiona a possibilidade de excluir pré-formismos sem recair em posições epistemológicas controversas.

A postulação daquelas linguagens, ou "processos cognitivos básicos", resulta em um desdobramento da gramática gerativa chomskyana: além da evidência de sistemas paralelos em lugar de uma visão unitária do processo de linguagem, um estado inicial $\left(\mathrm{S}_{0}\right)$ é admitido para além das relações gramaticais, envolvendo como se viu a ação e sua conseqüente virtualização na lógica e, por extensão, na matemática.

De importância mais imediata ao campo de ensino-aprendizagem, Seminerio enfatiza o uso "sistemático" das habilidades metacognitivas do aprendiz, cabendo ao professor papel ativo na veiculação e reflexão das regras generativas (ou "metaregras") - não propriamente inconscientes (cognized) como para Chomsky - cuja apreensão deve conduzir a um salto metaprocessual de parte daquele primeiro. Tal orientação representa segundo o autor uma proposta inovadora quer frente ao método dito tradicional de ensino, em que os alunos figuram como receptores passivos de informação pronta, quer frente ao ideal de liberdade do educando defendido pela Escola Nova e melhor fundamentado na posição construtivista. (SEMINERIO et al., 1997).

A formulação da técnica psicopedagógica da "elaboração dirigida" remete, em breve, ao conceito de modelação que Bandura (1977) descreve em sua teoria da aprendizagem social, ao romper com as teorias clássicas da aprendizagem 
comportamentista e reconhecer importância central à observação e imitação de modelos exteriores de conduta, que se tornam aprendidos por uma soma de fatores cognitivos, motores e motivacionais. "Trata-se", segundo Seminerio (1995a), "de uma variante do comportamento vicário", uma vez que a estocagem mnêmica de modelos comporta um sistema de regras primitivas que podem ser aplicadas futuramente, reforçadas pela própria percepção do domínio de novas situações.

Finalmente, o Laboratório de Metacognição, pesquisa coordenada por muitos anos pelo Professor Franco Lo Presti Seminerio no Instituto de Psicologia da UFRJ, obteve resultados expressivos no uso daquela técnica junto ao desenvolvimento do raciocínio lógico infantil, favorecendo a transição de crianças, mediante atividades lúdicas cuidadosamente planejadas, a um domínio recursivo (generalizante) das operações mentais de classificação/inclusão, seriação e conservação. Em seguida, outras investigações em equipe ou individuais têm se revelado eficazes quanto à aprendizagem (metacognitiva) de conceitos matemáticos iniciais, com base em tais operações, e ainda acerca do campo conceitual fracionário (CHAHON, 1999).

A competência em representar e resolver problemas aritméticos verbais, por sua vez, possui um valor pedagógico especial enquanto atividade catalisadora do pensamento matemático do aprendiz (CARPENTER et al., 1993) ou, segundo Vergnaud (apud FAYOL, 1996), tornando mais evidente a lacuna freqüentemente desapercebida entre as construções lógicas precoces da criança seus teoremas em ato e os "invariantes relacionais" transmitidos pela via escolar.

\section{O USO DA METACOGNiÇão EM SALA de AULA:} BREVE DESCRIÇÃO DE INTERVENÇÃO EMPÍRICA

Não cabendo neste artigo relatar pormenorizadamente a experimentação conduzida pelo autor, produto das diversas referências que aqui se buscou sintetizar, algumas observações podem ser feitas a fim de ilustrar o vínculo entre os temas centrais apresentados: a metacognição enquanto conceito-chave de uma técnica psicopedagógica inovadora, e o campo da resolução de problemas aritméticos verbais, cuja aprendizagem formal é também ao seu modo paradigmática quanto aos princípios educacionais subjacentes.

Em resumo, as provas diagnósticas individuais em torno de situações-problema de adição e subtração incluíram enunciados redigidos de modo convencional e cujo grau de dificuldade foi estabelecido com base em testagem prévia. Foram ainda elaborados itens complementares, inspirados na literatura revista, na tentativa de avaliar a compreensão lingüística do enunciado (tarefa de completar história com questão final apropriada) e a "sensibilidade semântica" da criança para com sua estrutura (tarefa de verificar a impossibilidade de resolver problemas mal formulados). Havia finalmente material auxiliar disponível, beneficiando a codificação pelo experimentador das estratégias de resolução empregadas (materiais, verbais ou mentais). 
As provas envolvendo situações mais simples de multiplicação e divisão seguiram padrão semelhante, e novamente foi adaptada tarefa complementar que, amparada no método clínico piagetiano, torna possível graduar as respostas de acordo com a emergência de um pensamento multiplicativo que elas revelam.

Por sua vez, as atividades de treinamento, junto a pequenos grupos de crianças, foram elaboradas na forma de roteiros flexíveis quanto ao número de sessões necessárias para cumprir objetivos específicos. Os "jogos" (ao menos o autor se esforçou em emprestar um contexto lúdico motivador às atividades) de adição/ subtração se ocuparam em transmitir (via modelação) as características estruturais distintivas dos problemas e as alterações decorrentes em especial da posição da incógnita: além de desenhos esquemáticos, procurou-se estimular representações escritas individuais e valorizar diferentes estratégias de resolução. Em sessões ditas de reforço, erros mais freqüentes cometidos na avaliação precedente aos jogos (por exemplo, erros de cálculo, de operação, ou quando a criança dá como resposta equivocada quantidade presente no enunciado) e, ainda, ressaltar a possibilidade de diferentes perguntas para um mesmo problema, ou inversamente.

Quanto à multiplicação/divisão, as atividades iniciais focalizaram competências como a construção de conjuntos equivalentes combinando diferentes proporções um-para-muitos, e a explicitação da relação inversa cota-número de partes no interior de situações concretas de divisão, adaptando mais uma vez experimentos que a literatura oferece. Somente em sessões finais é que foram introduzidas sentenças de tipo escolar e outras enriquecidas, tendo em vista dificuldades identificadas anteriormente, a fim de explorar a utilização de diferentes estratégias de contagem.

A nova testagem posterior ao treinamento evidenciou resultados quantitativamente tímidos, justificados em parte pela brevidade do mesmo. Embora nos primeiros encontros tratando de umas e outras operações a assimilação dos conteúdos e regras parecesse relativamente simples, desde o treinamento foram observadas dificuldades de vocabulário em problemas de comparação e divisão (notadamente do tipo "partitivo-combinação"), e os registros escritos um tanto pobres da maioria das crianças não deixou de refletir certa "urgência" em dar conta de objetivos muito amplos. Para além do aumento do número de respostas corretas, enfim, a análise das estratégias e erros mostrou um crescimento próximo, se bem que algo diferenciado, dos grupos submetidos ou não à experimentação.

\section{ComentáRios FinAis}

Nos termos do Laboratório de Metacognição, a elaboração dirigida visa à instalação de um raciocínio de segunda ordem, isto é, à generalização de uma atitude investigativa ou reflexiva que caracteriza o uso intencional das habilidades cognitivas do aprendiz. Além das áreas mencionadas do raciocínio lógico infantil (quarta linguagem-código: " $\mathrm{L}_{4}$ ") e do ensino-aprendizagem de conceitos matemáticos iniciais, outras investigações fundamentais ao projeto voltam-se ao desenvolvimento do vocabulário (" $\mathrm{L}_{2}$ "), do imaginário cognitivo (" $\mathrm{L}_{3}$ ") e à área da alfabetização infantil. 
Considerados os avanços assim obtidos nos muitos anos de funcionamento do Laboratório, vale recordar a advertência encontrada em Paula, acerca do desenvolvimento das habilidades de leitura, de que a atenção às funções metacognitivas na formulação de currículos escolares não deve ser confundida com uma panacéia a todos os problemas associados ao fracasso escolar, mas vista como um fator entre outros e não necessariamente favorável em todos os momentos da aprendizagem.

No domínio da educação matemática, a epistemologia genética serve de fundamento crítico por sua vez à precocidade com que muitos conceitos são transmitidos à criança, assim como à metodologia usual, que tende a negligenciar as habilidades infantis na resolução de problemas envolvendo cálculos aritméticos (KAMII, 1993).

Em resposta a tais observações gerais de grande importância, cumpre em primeiro lugar sugerir a leitura cuidadosa e direta dos escritos do Professor Franco Lo Presti Seminerio, sem o que a falsa interpretação daquela "panacéia" poderia de fato emergir, do caráter inovador que é associado (diga-se, de modo teoricamente claro) ao conceito de metacognição em sua vertente psicopedagógica.

Já quanto à aplicação da técnica em área de ensino formal como a Matemática, os obstáculos à experimentação parecem aumentar de saída pela influência do ensino prévio e concomitante a modelo de treinamento como o descrito acima, inclusive no âmbito motivacional. E como a menção às idéias hoje bem conhecidas de Kamii permite entrever, as dificuldades em mensurar apropriadamente o que é "ensinável" a crianças de determinada idade e classe escolar evidenciam a relevância de pesquisas contínuas de cunho interdisciplinar.

Em especial, o tema crescentemente pesquisado da resolução de problemas pode ser identificado como paradigmático quer em termos do exame das capacidades cognitivas do aprendiz (e, mais raramente, do professor), quer enquanto tema privilegiado de campo de estudos que hoje se denomina "psicologia da educação matemática", em função de justaposição promovida entre as três disciplinas.

\section{Nota}

1 Este artigo procura resumir aspectos teóricos críticos da Tese de Doutorado em Psicologia do autor, intitulada "A Metacognição e a Resolução de Problemas Aritméticos Verbais em Sala de Aula: pesquisa e intervenção", realizada sob a orientação do Professor Franco Lo Presti Seminerio, defendida em 01/2004 no Instituto de Psicologia da UFRJ. 


\section{REFERÊNCIAS}

BANDURA, A. Social learning theory. New Jersey: Prentice-Hall, 1977.

BELL, A. et al. Children's performance on multiplicative word problems: elements of a descriptive theory. Journal for Research in Mathematics Education, [S.L], v. 20, n. 5, p. 434-449, Nov. 1989.

BRITO, M. R. F. Este problema é difícil porque não é de escola!: a compreensão e a solução de problemas verbais por crianças da escola fundamental. XXIX Revista da Sociedade Brasileira de Psicologia, São Paulo, v. 8, n. 1, p. 93-109, 2000. Temas em Psicologia da SBP.

BRUNER, J. S. A cultura da educação. Porto Alegre: Artmed, 2001.

. Uma nova teoria da aprendizagem. 4. ed. Rio de Janeiro: Bloch, 1976.

CARPENTER, T. P., et al. Models of problem solving: a study of kindergarden children's problem-solving processes. Journal for Research in Mathematics Education, [S.L], v. 24, n. 5, p. 428-441, 1993.

CHAHON, M. O Uso da metacognição no ensino fundamental da matemática: uma proposta de intervenção. Arquivos Brasileiros de Psicologia, Rio de Janeiro, v. 51, n. 3, p. 52-59, jan./mar. 1999.

CUMMINS, et al. The role of understanding in solving word problems. Cognitive Psychology, [S.1.], v. 20, n. 4, p. 405-438, 1988.

DAVIDSON, J. E.; DEUSER, R.; STERNBERG, R. J. The role of metacognition in problem solving. In METCALFE, J.; SHIMAMURA, A. P. (Org.). Metacognition: knowing about knowing. Boston: MIT Press, 1996. p. 207-226.

FAYOL, M. A criança e o número: da contagem à resolução de problemas. Porto Alegre: Artes Médicas, 1996.

FINI, L. D. T. Aritmética no ensino fundamental: análise psicopedagógica. In: SISTO, F. F.; MARTINELLI, S. C. (Org.). Dificuldades de aprendizagem no contexto psicopedagógico. Petrópolis:Vozes, 2001. p. 60-78.

FLAVELL, J.H.; MILLER, P. H ; MILLER, S. A. Desenvolvimento cognitivo. 3. ed. Porto Alegre: Artes Médicas, 1999.

KAMII, C. A criança e o número: implicações da teoria de Piaget para a atuação junto a escolares de 4 a 6 anos. 10. ed. Campinas: Papirus, 1989.

. Aritmética: novas perspectivas - implicações da teoria de Piaget. 2. ed. Campinas: Papirus, 1993.

KOUBA, V. L. Children's solution strategies for equivalent set multiplication and division word problems. Journal for Research in Mathematics Education, [S.L], v. 20, n. 2 , p. $147-158,1989$. 
MAYER, R. E. A Capacidade para a matemática. In: STERNBERG, R. (Org.). As capacidades intelectuais humanas: uma abordagem em processamento de informações. Porto Alegre: Artes Médicas. 1992. p. 144-168.

NUNES, T.; BRYANT, P. Crianças fazendo matemática. Porto Alegre: Artes Médicas, 1997.

ONUCHIC, L. R. Ensino-aprendizagem de matemática através da resolução de problemas. In: BICUDO, M. A. V. (Org.). Pesquisa em educação matemática: concepções e perspectivas. São Paulo: UNESP. 1999. p. 199-218.

PAULA, F. V. de. Conhecimento metacognitivo de Crianças de $3^{a}$ série que apresentam dificuldades na aquisição de leitura. 2001. Dissertação (Mestrado) Instituto de Psicologia, Universidade de São Paulo, Campinas, SP, 2001.

SELVA, A. C. V. Discutindo o uso de materiais concretos na resolução de problemas de divisão. In: SCHLIEMANN, A. D; CARRAHER, D. W. (Org.). A compreensão de conceitos aritméticos: ensino e pesquisa. Campinas: Papirus, 1998. p. 95-119.

SEMINERIO, F. L. P. A Metacognição e seus usos: um mecanismo geral de desenvolvimento cognitivo. Arquivos Brasileiros de Psicologia, Rio de Janeiro, v. 47, n. 3, p. 3-29, abr./jun. 1995a.

. Códigos morfogenéticos da Cognição. Arquivos Brasileiros de Psicologia, Rio de Janeiro, v. 47, n. 1, p. 3-45, jan./mar. 1995b.

. O construtivismo e os limites do pré-formismo. Arquivos Brasileiros de Psicologia, Rio de Janeiro, v.36, n. 4, p. 3-11, out./dez. 1984.

. Origem dos universais da cognição. Arquivos Brasileiros de Psicologia, Rio de Janeiro, v. 46, n. 3-4, p. 3-17, jul./dez. 1994.

SEMINERIO, F. L. P. et al. Metacognição: um novo paradigma. Arquivos Brasileiros de Psicologia, Rio de Janeiro, v. 51, n. 1, p. 110-126, jan./mar. 1999.

. Novos rumos na Psicologia e na Pedagogia: metacognição: uma nova opção. Arquivos Brasileiros de Psicologia, Rio de Janeiro, v. 49, n. 3, p. 5-22, jul./set. 1997.

SILVA, J. J. Filosofia da matemática e filosofia da educação matemática. In: BICUDO, M.A.V. (Org.). Pesquisa em educação matemática: concepções e perspectivas. São Paulo: UNESP, 1999. p. 45-58.

SMOLE, K .S.; DINIZ, M. I. (Org.). Ler, escrever e resolver problemas: habilidades básicas para aprender matemática. Porto Alegre: Artmed, 2001.

STERNBERG, R . J. Psicologia cognitiva. Porto Alegre: Artes Médicas Sul, 2000.

VASCONCELOS, L. Problemas de adição e subtração: modelos teóricos e práticas de ensino. In: SCHLIEMANN, A .D.; CARRAHER, D. W. (Org.). A compreensão de conceitos aritméticos: ensino e pesquisa. Campinas: Papirus, 1998. p. 53-72. 
VERGNAUD, G. Psicologia do Desenvolvimento Cognitivo e Didática das Matemáticas-um exemplo: as estruturas aditivas. Análise Psicológica, [S.1.], v. 1, n. 5, p. 75-90, 1986.

WOOD, D. Como as crianças crescem e aprendem. São Paulo: Martins Fontes, 1996.

Recebido em: janeiro/2006.

Aceito em: maio/ 2006. 
